\title{
A line of zebrafish with development of abnormal spinal curvatures
}

\author{
Henry Tomasiewicz ${ }^{1 *}$, John Thometz ${ }^{2}$, XueCheng Liu², Channing Tassone², Paula North² \\ From 11th International Conference on Conservative Management of Spinal Deformities - SOSORT 2014 \\ Annual Meeting \\ Wiesbaden, Germany. 8-10 May 2014
}

\section{Background}

The lack of a good animal model system has hindered studying the etiology of idiopathic scoliosis. Recently, it has become clear that several fish species appear to exhibit spinal curvatures.

\section{Aims}

To determine if the affected tissue in a line of zebrafish with spinal deformities resembles the pathology observed in pediatric populations with scoliosis.

\section{Design}

This case series study was approved by IACUC.

\section{Methods}

Potential founder fish with spinal curvatures were outcrossed with a wild type zebrafish line $(\mathrm{AB})$ and the resulting siblings (F1 generation) crossed and the offspring (F2 generation) examined for signs of spinal curvature beginning at 14 days post fertilization (dpf). Spinal curvatures of the affected fish were visualized using either a Faxitron or by Alizarian red staining of the skeletons and the curvature measured from the resulting images in the thoracic, thoracolumbar, or lumbar regions. Affected and normal zebrafish were fixed, embedded, section and stained with hemotoxylin and eosin.

\section{Results}

The degrees of curvatures ranged from $18^{\circ}$ to $40^{\circ}$ Histological data demonstrated structural changes as compared to normal fish spine. Out of 212 individuals in the F2 generation 28 , or $13.2 \%$, were observed to have spinal deformities at $21 \mathrm{dpf}$. Importantly, we did not observe spinal deformities in the F1 generation fish and similar age wild type fish, indicating the observed spinal deformities were due to a recessive mutation(s).

\section{Conclusion}

An increase of scoliosis in family members and the occurrence of abnormal spinal curvatures in twins suggest a polygenetic inheritance pattern. We have noticed several fish in our zebrafish colony with spinal curvatures reminiscent of human idiopathic scoliosis. We suspect that "scoliosis" in these zebrafish results from mutation(s) in the zebrafish genome. And these fish can be the source of a zebrafish line in which the offspring exhibit a predictable frequency of scoliosis which can be used to study the etiology and progression of scoliosis.

\section{Authors' details}

'University of Wisconsin-Milwaukee, Milwaukee, WI, USA. ${ }^{2}$ Medical College of WI, Milwaukee, WI, USA.

Published: 4 December 2014

doi:10.1186/1748-7161-9-S1-044

Cite this article as: Tomasiewicz et al: A line of zebrafish with

development of abnormal spinal curvatures. Scoliosis 2014 9(Suppl 1):044.

${ }^{1}$ University of Wisconsin-Milwaukee, Milwaukee, WI, USA

Full list of author information is available at the end of the article

(c) 2014 Tomasiewicz et al; licensee BioMed Central Ltd. This is an Open Access article distributed under the terms of the Creative 